\title{
Certification and Quantification of Multilevel Quantum Coherence
}

\author{
Martin Ringbauer, ${ }^{1,2,3,6,8}$ Thomas R. Bromley, ${ }^{4}$ Marco Cianciaruso, ${ }^{4}$ Ludovico Lami, ${ }^{4}$ W. Y. Sarah Lau, ${ }^{2,3}$ \\ Gerardo Adesso, ${ }^{4, *}$ Andrew G. White, ${ }^{2,3}$ Alessandro Fedrizzi, ${ }^{1, \dagger}$ and Marco Piani ${ }^{5, \$}$ \\ ${ }^{1}$ Institute of Photonics and Quantum Sciences, School of Engineering and Physical Sciences, \\ Heriot-Watt University, Edinburgh EH14 4AS, United Kingdom \\ ${ }^{2}$ Centre for Engineered Quantum Systems, School of Mathematics and Physics, \\ University of Queensland, Brisbane, Queensland 4072, Australia \\ ${ }^{3}$ Centre for Quantum Computation and Communication Technology, School of Mathematics and Physics, \\ University of Queensland, Brisbane, Queensland 4072, Australia \\ ${ }^{4}$ Centre for the Mathematics and Theoretical Physics of Quantum Non-Equilibrium Systems (CQNE), \\ School of Mathematical Sciences, The University of Nottingham, \\ University Park, Nottingham NG7 2RD, United Kingdom \\ ${ }^{5}$ SUPA and Department of Physics, University of Strathclyde, Glasgow G4 ONG, United Kingdom \\ ${ }^{6}$ Institut für Experimentalphysik, Universität Innsbruck, 6020 Innsbruck, Austria
}

(Received 2 May 2018; revised manuscript received 15 August 2018; published 10 October 2018)

\begin{abstract}
Quantum coherence, present whenever a quantum system exists in a superposition of multiple classically distinct states, marks one of the fundamental departures from classical physics. Quantum coherence has recently been investigated rigorously within a resource-theoretic formalism. However, the finer-grained notion of multilevel coherence, which explicitly takes into account the number of superposed classical states, has remained relatively unexplored. A comprehensive analysis of multilevel coherence, which acts as the singleparty analogue to multipartite entanglement, is essential for understanding natural quantum processes as well as for gauging the performance of quantum technologies. Here, we develop the theoretical and experimental groundwork for characterizing and quantifying multilevel coherence. We prove that nontrivial levels of purity are required for multilevel coherence, as there is a ball of states around the maximally mixed state that do not exhibit multilevel coherence in any basis. We provide a simple, necessary, and sufficient analytical criterion to verify the presence of multilevel coherence, which leads to a complete classification of multilevel coherence for three-level systems. We present the robustness of multilevel coherence, a bona fide quantifier, which we show to be numerically computable via semidefinite programming and experimentally accessible via multilevel coherence witnesses, which we introduce and characterize. We further verify and lower bound the robustness of multilevel coherence by performing a semi-device-independent phase discrimination task, which is implemented experimentally with four-level quantum probes in a photonic setup. Our results contribute to understanding the operational relevance of genuine multilevel coherence, also by demonstrating the key role it plays in enhanced phase discrimination-a primitive for quantum communication and metrology — and suggest new ways to reliably and effectively test the quantum behavior of physical systems.
\end{abstract}

DOI: 10.1103/PhysRevX.8.041007

\section{INTRODUCTION}

Quantum coherence manifests whenever a quantum system is in a superposition of classically distinct states,

\footnotetext{
*gerardo.adesso@nottingham.ac.uk

†a.fedrizzi@hw.ac.uk

*marcopiani@gmail.com

${ }^{\S}$ martin.ringbauer@uibk.ac.at
}

Published by the American Physical Society under the terms of the Creative Commons Attribution 4.0 International license. Further distribution of this work must maintain attribution to the author(s) and the published article's title, journal citation, and DOI.
Subject Areas: Photonics, Quantum Physics,

Quantum Information such as different energy levels or spin directions. Formally, a quantum state displays coherence (For brevity, we will omit the qualifier "quantum" in the following.) whenever it is described by a density matrix that is not diagonal with respect to the relevant orthogonal basis of classical states [1]. In this sense, coherence underpins virtually all quantum phenomena, yet it has only recently been characterized formally [2-4]. Coherence is now recognized as a fully fledged resource and studied in the general framework of quantum resource theories [1,5-7]. This has led to a menagerie of possible ways to quantify coherence in a quantum system [1,3,8-18], along with an intense analysis of how coherence plays a role in fundamental physics, 
e.g., in quantum thermodynamics $[19,20]$, and in operational tasks relevant to quantum technologies, including quantum algorithms and quantum metrology [8,9,16,18,21-24].

Despite a great deal of recent progress, however, the majority of current literature focuses on a rather coarsegrained description of coherence, which is ultimately insufficient to reach a complete understanding of the fundamental role of quantum superposition in the aforementioned tasks. To overcome such limitations, one needs to take into consideration the number of classical states in coherent superposition - contrasted with the simpler question of whether any nontrivial superposition exists-which gives rise to the concept of multilevel quantum coherence [25-27]. Similarly to the existence of different degrees of entanglement in multipartite systems, going well beyond the mere presence or absence of entanglement and corresponding to different capabilities in quantum technologies $[28,29]$, one can then identify and study a rich structure for multilevel coherence. Deciphering this structure can yield a tangible impact on many areas of physics, such as condensed matter, statistical mechanics, and transfer phenomena in many-body systems [25,30-32]. For example, for understanding the role of coherence in the function of complex biological molecules, such as those found in light harvesting, it will be crucial to differentiate between pairwise coherence among the various sites in the molecule and genuine multilevel coherence across many sites [32-35]. In quantum computation, large superpositions of computational basis states need to be generated, and effective benchmarking of such devices requires proper tools to certify and quantify multilevel coherence.

Recent works have presented initial approaches to measuring the amount of multilevel coherence [36], as well as schemes to convert it into bipartite and genuine multipartite entanglement, enabling the fruitful use of entanglement theory tools to study coherence itself $[27,37,38]$. Nonetheless, an all-inclusive systematic framework for the characterization, certification, and quantification of multilevel coherence is still lacking.

Here, we construct and present such a theoretical framework for multilevel coherence and apply it to the experimental verification and quantification of multilevel coherence in a quantum optical setting. We begin by developing a resource theory of multilevel coherence, in particular, providing a new characterization of the sets of multilevel coherence-free states [see Fig. 1(a)] and free operations, rigorously unfolding the hierarchy of multilevel coherence. We present analytical criteria for multilevel coherence, which lead to a complete classification of multilevel coherence for three-level systems and which establish lower bounds on the purity required to exhibit multilevel coherence. We then formalize the robustness of multilevel coherence and show that it is an efficiently computable measure, which is experimentally accessible through multilevel coherence witnesses. Using photonic
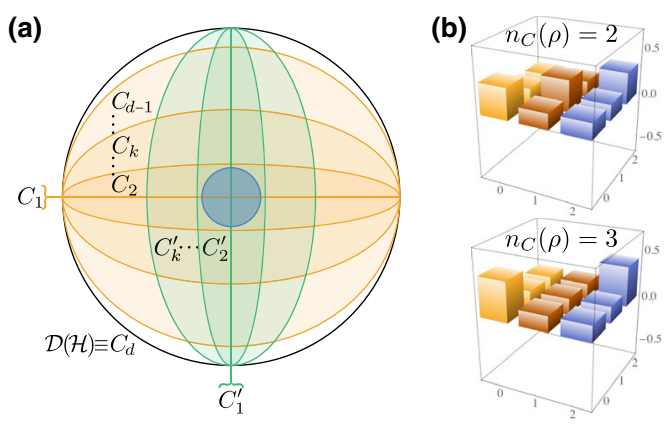

FIG. 1. Hierarchy of multilevel coherence. (a) The set of states $\mathcal{D}(\mathcal{H})$ (outer circle) of a $d$-dimensional quantum system, which can be structured according to coherence number into the convex sets $C_{k}$ (orange shading) with $C_{1} \subset C_{2} \subset \ldots \subset C_{d}=\mathcal{D}(\mathcal{H})$. Note that a different choice of classical basis leads to a different hierarchy of sets $C_{k}^{\prime}$ (green shading). However, as shown in the Supplemental Material [41], irrespective of the classical basis, there is a finite volume ball within $C_{2}$ (blue inner circle). This implies that, while almost all states exhibit some form of coherence, achieving genuine multilevel coherence is instead nontrivial and requires the state to be sufficiently far from the maximally mixed state. (b) Real part of the density matrix of two example three-dimensional quantum states with equal off-diagonal elements yet different multilevel-coherence properties. The upper state is a mixture of two level-coherent states of the form $\frac{1}{3}\left(\left|\psi_{0,1}\right\rangle\left\langle\psi_{0,1}|+| \psi_{0,2}\right\rangle\left\langle\psi_{0,2}|+| \psi_{1,2}\right\rangle\left\langle\psi_{1,2}\right|\right)$, where $\left|\psi_{i, j}\right\rangle=\frac{1}{\sqrt{2}}(|i\rangle+|j\rangle)$, and thus has coherence number $n_{C}(\rho)=2$. The lower state is a mixture of the maximally coherent state $(|0\rangle+|1\rangle+|2\rangle) / \sqrt{3}$ with weight $1 / 2$ and of the incoherent-basis states $|0\rangle$ and $|2\rangle$, each with weight $1 / 4$. Every pure-state decomposition of the lower state must contain a superposition of all $|0\rangle,|1\rangle,|2\rangle$, as it can be verified by numerically calculating the robustness of three-level coherence $R_{C_{2}} \approx 0.0361$ [see Eq. (2)] or by using the comparison matrix criterion of Sec. II C. Hence, although both states exhibit the same off-diagonal elements, only the lower state has genuine multilevel coherence, requiring experimental control that is coherent across multiple levels. This exemplifies the fine-grained classification of coherence and of experimental capabilities that studying multilevel coherence provides.

four-dimensional systems, we demonstrate how to quantify, witness, and bound multilevel coherence experimentally. We prove that multilevel coherence, quantified by our robustness measure, has a natural operational interpretation as a fundamental resource for quantum phase discrimination $[8,9]$, a cornerstone task for quantum metrology and communication technologies $[39,40]$. In turn, we show how to exploit this task to experimentally lower bound the robustness of multilevel coherence of an unknown quantum state in a semi-device-independent manner.

Our results yield a significant step forward in the theoretical and experimental quest for the full characterization of (multilevel) coherence as a core feature of quantum systems, and they provide a practically useful toolbox for the performance assessment of upcoming quantum technologies exploiting multilevel coherence as a resource. 


\section{RESULTS}

\section{A. Resource theory of multilevel coherence}

We generalize the recently formalized resource theory of coherence [1] to the notion of multilevel coherence. We remind the reader that the general structure of a resource theory contains three main ingredients, which we present below: a set of free states, which do not contain the resource; a set of free operations, which are quantum operations that cannot create the resource; and a measure of the resource.

\section{Multilevel coherence-free quantum states}

Consider a $d$-dimensional quantum system with Hilbert space $\mathcal{H} \simeq \mathbb{C}^{d}$, spanned by an orthonormal basis $\{|i\rangle\}_{i=1}^{d}$, with respect to which we measure quantum coherence. The choice of classical basis is typically fixed to correspond to the eigenstates of a physically relevant observable like the system Hamiltonian. Any pure state $|\psi\rangle \in \mathcal{H}$ can be written in this basis as $|\psi\rangle=\sum_{i=1}^{d} c_{i}|i\rangle$, with $\sum_{i=1}^{d}\left|c_{i}\right|^{2}=1$. The state $|\psi\rangle$ exhibits some quantum coherence with respect to the basis $\{|i\rangle\}_{i=1}^{d}$ whenever at least two of the coefficients $c_{i}$ are nonzero [1]. The multilevel nature of coherence is revealed by the number of nonzero coefficients $c_{i}$, the so-called coherence rank $r_{C}[25,33]$. We say that a state $|\psi\rangle$ has coherence rank $r_{C}(|\psi\rangle)=k$ if exactly $k$ of the coefficients $c_{i}$ are nonzero. The notion of coherence rank thereby provides a fine-grained account of the quantum coherence of $|\psi\rangle$, as compared to merely establishing the presence of some coherence.

To generalize multilevel coherence to mixed states $\rho \in$ $\mathcal{D}(\mathcal{H})$, we define the sets $C_{k} \subseteq \mathcal{D}(\mathcal{H})$ with $k \in\{1, \ldots, d\}$, given by all probabilistic mixtures of pure density operators $|\psi\rangle\langle\psi|$ with a coherence rank of at most $k$,

$$
C_{k}:=\operatorname{conv}\left\{|\psi\rangle\langle\psi|: r_{C}(|\psi\rangle) \leq k\right\},
$$

where conv stands for "convex hull." Here, $C_{1}$ is the set of fully incoherent states, given by density matrices that are diagonal in the classical basis, while $C_{d} \equiv \mathcal{D}(\mathcal{H})$ is the set of all states. The intermediate sets obey the strict hierarchy, $C_{1} \subset C_{2} \subset \ldots \subset C_{d}$ [see Fig. 1(a)] and are the free states in the resource theory of multilevel coherence; e.g., $C_{k}$ is the set of $(k+1)$-level coherence-free states.

For a general mixed state, one defines the coherence number $n_{C}$ [36-38], such that a state $\rho \in \mathcal{D}(\mathcal{H})$ has a coherence number $n_{C}(\rho)=k$ if $\rho \in C_{k}$ and $\rho \notin C_{k-1}$ (for consistency, we set $C_{0}=\varnothing$ ). This parallels the notions of Schmidt number [42] and entanglement depth [43] in entanglement theory. A state with coherence number $n_{C}(\rho)=k$ can be decomposed into (at most $d^{2}$ ) pure states with coherence rank of at most $k$, while every such decomposition must contain at least one state with coherence rank of at least $k$. A state with $n_{C}(\rho)=k$ is said to exhibit genuine $k$-level coherence, distinguishing it from states that may display coherence between several pairs of levels - potentially even between all such pairs-yet can be prepared as mixtures of pure states with relatively lowerlevel coherence; see Fig. 1(b). In an experiment, the presence of multilevel coherence proves the ability to coherently manipulate a physical system across many of its levels, much in the same way that the creation of states with large entanglement depth provides a certification of the coherent control over several systems.

Note that a state may, at the same time, display large toutcourt coherence but have vanishing higher-level coherence. This is the case, for example, for a superposition of $d-1$ basis elements, like $|\psi\rangle=\sqrt{1 /(d-1)} \sum_{i=2}^{d}|i\rangle$, which does not display $d$-level coherence despite being highly coherent. On the other hand, a pure state may be arbitrarily close to one of the elements of the incoherent basis yet display nonzero genuine multilevel coherence for all $k$. This is the case, for example, for the state $|\phi\rangle=$ $\sqrt{1-\epsilon}|1\rangle+\sqrt{\epsilon /(d-1)} \sum_{i=2}^{d}|i\rangle$ for small $\epsilon$. It should be clear that the above multilevel classification provides a much finer description of the coherence properties of quantum systems but that it is also important to elevate such a finer qualitative classification to a finer quantitative description, as we do in the following.

\section{Multilevel coherence-free operations and $k$-decohering operations}

The second ingredient in the resource theory of multilevel coherence is the set of operations that do not create multilevel coherence. A general quantum operation $\Lambda$ is described by a linear completely positive and trace-preserving (CPTP) map, whose action on a state $\rho$ can be written as $\Lambda(\rho)=\sum_{i} K_{i} \rho K_{i}^{\dagger}$, in terms of (nonunique) Kraus operators $\left\{K_{i}\right\}$ with $\sum_{i} K_{i}^{\dagger} K_{i}=\mathbb{I}$ [44]. For any map $\Lambda$ and any set $S$ of states, we denote $\Lambda(S):=\{\Lambda(\rho): \rho \in S\}$. Generalizing the formalism introduced for standard coherence [1-3], we refer to a CPTP map $\Lambda$ as a $k$-coherence-preserving operation if it cannot increase the coherence level, i.e., $\Lambda\left(C_{k}\right) \subseteq C_{k}$. An important subset of these are the $k$-incoherent operations, which are all CPTP maps for which there exists a set of Kraus operators $\left\{K_{i}\right\}$ such that $K_{i} \rho K_{i}^{\dagger} / \operatorname{Tr}\left(K_{i} \rho K_{i}^{\dagger}\right) \in C_{k}$ for any $\rho \in C_{k}$ and all $i$. Note that the (fully) incoherent operations from the resource theory of coherence correspond to $k=1$. In the Supplemental Material [41], we prove that fully incoherent operations are also $k$-incoherent operations for all $k$, and we further define the notion of $k$-decohering maps as those that destroy multilevel coherence: An operation $\Lambda$ is $k$ decohering if $\Lambda(\mathcal{D}(\mathcal{H})) \subseteq C_{k}$. In particular, we introduce a family of maps that generalize the fully decohering map $\Delta[X]=\sum_{i=1}^{d}|i\rangle\langle i|X| i\rangle\langle i|$, which is such that $\Delta(\mathcal{D}(\mathcal{H})) \equiv C_{1}$.

\section{Measure of multilevel coherence}

The final ingredient for the resource theory of multilevel coherence is a well-defined measure. Very few quantifiers 
of such a resource have been suggested, and those that exist lack a clear operational interpretation [36,38]. Furthermore, many of the quantifiers of coherence, such as the intuitive $l_{1}$ norm of coherence, which measures the off-diagonal contribution to the density matrix, fail to capture the intricate structure of multilevel coherence, as indicated in Fig. 1(b). Here, we introduce the robustness of multilevel coherence (RMC) $R_{C_{k}}(\rho)$ as a bona fide measure that is directly accessible experimentally and efficient to compute for any density matrix. The robustness of $(k+1)$-level coherence can be understood as the minimal amount of noise that has to be added to a state to destroy all $(k+1)$-level coherence, defined as

$$
R_{C_{k}}(\rho):=\inf _{\tau \in \mathcal{D}(\mathcal{H})}\left\{s \geq 0: \frac{\rho+s \tau}{1+s} \in C_{k}\right\} .
$$

This measure generalizes the recently introduced robustness of coherence [8,9] [corresponding to $R_{C_{1}}(\rho)$ ] to provide full sensitivity to the various levels of multilevel coherence. As a special case of the general notion of robustness of a quantum resource [45-52], the quantities $R_{C_{k}}$ are known to be valid resource-theoretic measures $[1,28]$, satisfying non-negativity, convexity, and monotonicity on average with respect to stochastic free operations $[8,9,45,46,53]$. The latter means for any $\rho$ that $R_{C_{k}}(\rho) \geq \sum_{i} p_{i} R_{C_{k}}\left(\rho_{i}\right)$ for all $k$-incoherent operations with Kraus operators $\left\{K_{i}\right\}$ such that $p_{i}=\operatorname{Tr}\left(K_{i} \rho K_{i}^{\dagger}\right)$ and $\rho_{i}=K_{i} \rho K_{i}^{\dagger} / p_{i}$. Since (fully) incoherent operations are $k$ incoherent for any $k$, the RMC also satisfies the strict monotonicity requirement for coherence measures $[1,3]$; see Supplemental Material [41].

Crucially, we find that the RMC can be posed as the solution of a semidefinite program (SDP) optimization problem [54-56]; see Supplemental Material [41]. A variety of algorithms exist to solve SDPs efficiently [55], meaning that the RMC may be computed efficiently for any $k$-in stark contrast to the robustness of entanglement $[45,46]$ where one has to deal with the subtleties of the characterization of the set of separable states [50]. For an arbitrary $d$-dimensional quantum state, we find that

$$
0 \leq R_{C_{k}}(\rho) \leq \frac{d}{k}-1 \quad \forall \rho \in \mathcal{D}(\mathcal{H})
$$

since any such state can be deterministically prepared using only (fully) incoherent operations [3] starting from the maximally coherent state $\left|\psi_{d}^{+}\right\rangle=d^{-1 / 2} \sum_{i=1}^{d}|i\rangle$, for which $R_{C_{k}}(\rho)=\frac{d}{k}-1$ (see Supplemental Material [41]).

\section{B. Experimental verification and quantification of multilevel coherence}

We apply our theoretic framework to an experiment that produces four-dimensional quantum states with varying degrees and levels of coherence using the setup in Fig. 2.

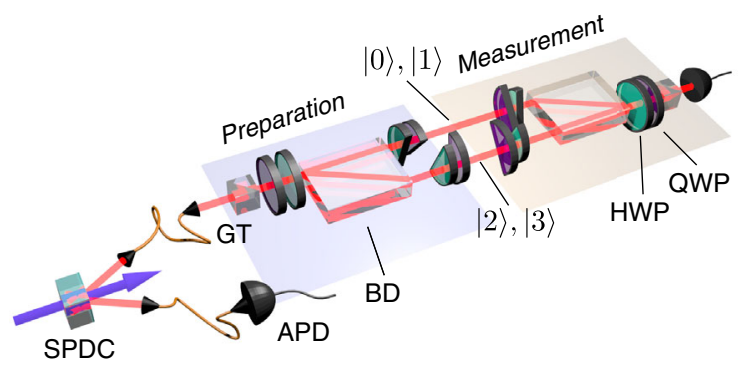

FIG. 2. Experimental setup for probing multilevel coherence in systems of dimension $d \leq 4$. Pairs of single photons are created via spontaneous parametric down-conversion (SPDC) in a $\beta$-Barium-borate (BBO) crystal, pumped at a wavelength of $410 \mathrm{~nm}$. The detection of one photon heralds the presence of the other, which is initialized in a horizontal polarization state by means of a Glan-Taylor polarizer (GT). A four-level quantum system is then prepared using polarization encoding in each of the two spatial modes created by a calcite beam displacer (BD). Three sets of half-wave plates (HWP) and quarter-wave plates (QWP) are used to control the amplitude and phase of the generated states. We prepare noisy maximally coherent states $\rho(p)$, Eq. (4), for several values of $p$ in dimension $d=4$, as detailed in (b). Arbitrary states can be prepared and measured in dimension $d \leq 4$ by manipulating only the corresponding subspaces.

We use heralded single photons at a rate of about $10^{4} \mathrm{~Hz}$, generated via spontaneous parametric down-conversion in a $\beta$-Barium borate crystal, pumped by a femtosecond pulsed laser at a wavelength of $410 \mathrm{~nm}$. We encode quantum information in the polarization and path degrees of freedom of these photons to prepare four-dimensional systems [57] with the basis states $|0\rangle=|H\rangle_{1},|1\rangle=|V\rangle_{1}$, $|2\rangle=|H\rangle_{2},|3\rangle=|V\rangle_{2}$, where $|p\rangle_{m}$ denotes a state of polarization $p$ in mode $m$. This dual encoding allows for high-precision preparation of arbitrary pure quantum states of any dimension $d \leq 4$ with an average fidelity of $\mathcal{F}=$ $0.997 \pm 0.002$ and purity of $\mathcal{P}=0.995 \pm 0.003$. An arbitrary mixed state $\rho$ can be engineered as a proper mixture, by preparing the states of a pure-state decomposition of $\rho$ for appropriate fractions of the total measurement time and tracing out the classical information about which preparation was implemented. Using the same technique, we can also subject the input states to arbitrary forms of noise.

Reversing the preparation stage of the setup allows us to implement arbitrary sharp projective measurements. Arbitrary generalized measurements [44] are correspondingly implemented as proper mixtures of a projective decomposition with an average fidelity of $\mathcal{F}=0.997 \pm$ 0.002 . By design, our experiment implements one measurement outcome at a time, which achieves superior precision through the use of a single fiber-coupling assembly [57], while reducing systematic bias. The whole experiment is characterized by a quantum process fidelity of $\mathcal{F}_{p}=0.9956 \pm 0.0002$, limited by the interferometric contrast of about 300:1. The latter is stable over the relevant timescales of the experiment due to the inherently 
stable interferometric design with common mode noise rejection for all but the piezo-driven rotational degrees of freedom of the second beam displacer. All data presented here were integrated over $20 \mathrm{~s}$ for each outcome, which is also much faster than the observed laser drifts on the order of hours. The main source of statistical uncertainties thus comes from the Poisson-distributed counting statistics. This has been taken into account through Monte Carlo resampling, with $10^{4}$ runs for tomographic measurements and $10^{5}$ runs for all other measurements. All experimental data presented in the figures and text throughout the paper are based on at least $10^{5}$ single photon counts and contain $5 \sigma$ equivalent statistical confidence intervals, which are with high confidence normal distributed unless otherwise stated.

\section{Test-bed family of states}

To illustrate the phenomenology of multilevel coherence, we consider a family of noisy maximally coherent states

$$
\rho(p)=(1-p) \frac{\mathbb{I}}{d}+p\left|\psi_{d}^{+}\right\rangle\left\langle\psi_{d}^{+}\right|,
$$

with $p \in[0,1]$ and finite dimension $d$. These states interpolate between the maximally mixed state $\mathbb{I} / d$ (for $p=0$ ) and the maximally coherent state $\left|\psi_{d}^{+}\right\rangle=$ $d^{-1 / 2} \sum_{i=1}^{d}|i\rangle$ (for $p=1$ ). For this class of states, the RMC can be evaluated analytically as (see Supplemental Material [41])

$$
R_{C_{k}}(\rho(p))=\max \left\{\frac{p(d-1)-(k-1)}{k}, 0\right\} .
$$

In particular, this implies that $\rho(p) \in C_{k}$ for $p \leq(k-$ $1 / d-1)$ and $\rho(p) \notin C_{k}$ for $p>(k-1 / d-1)$; see Supplemental Material [41]. The family of noisy maximally coherent states thus provides the ideal test bed for our investigation, spanning the full hierarchy of multilevel coherence (see Fig. 3). Using the setup of Fig. 2, we engineer noisy maximally coherent states $\rho(p)$ for $d=4$ and a variety of values of $p$. We then reconstruct the experimentally prepared states using maximum-likelihood quantum-state tomography and compute the robustness coherence for all $k$ by evaluating the corresponding SDP [see Eq. (S13) of the Supplemental Material [41]]. As illustrated in Fig. 4, this method produces very reliable results; however, it requires $d^{2}$ measurements and is thus experimentally infeasible already for medium-scale systems. In the following, we introduce and use multilevelcoherence witnesses and other techniques to overcome such a limitation.

\section{Conditions for genuine multilevel coherence}

Given a density matrix $\rho$, we need to decide whether $n_{C}(\rho)=1$, as, by definition, this happens if and only if $\rho$ is

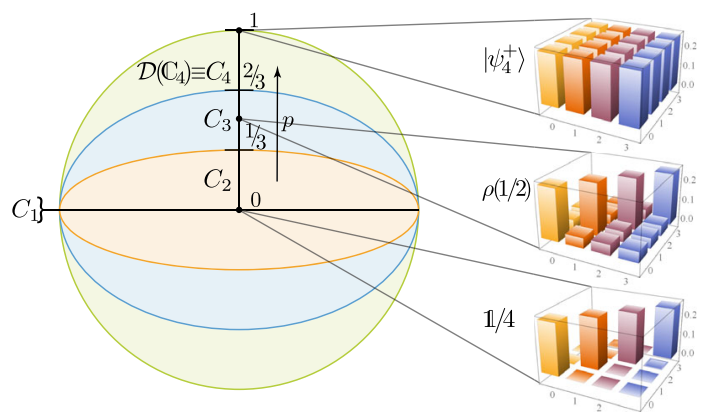

FIG. 3. Multilevel coherence in four-dimensional noisy maximally coherent states. With varying parameter $p \in[0,1]$, the coherence number of a four-dimensional, noisy maximally coherent state ranges from $n_{C}(\rho(p))=1$ for $p=0$, to $n_{C}(\rho(p))=2$ for $\left.p \in\right] 0, \frac{1}{3}$ ] (blue region), $n_{C}(\rho(p))=3$ for $p \in$ ]$\frac{1}{3}, \frac{2}{3}$ ] (orange region), and $n_{C}(\rho(p))=4$ for $\left.\left.p \in\right] \frac{2}{3}, 1\right]$ (green region). We use this color scheme throughout the paper to represent the three nontrivial levels of coherence in a fourdimensional system. On the right, we show examples of ideal density matrices for $p=1$ (top), $p=1 / 2$ (middle), and $p=0$ (bottom).

diagonal. While in Sec. II D we show how one can witness any multilevel coherence through the use of tailored multilevel-coherence witnesses, in this section we focus on simple analytical necessary and sufficient criteria for multilevel coherence. Such criteria also allow us to establish that all sets $C_{k}$, for $k \geq 2$, have nonzero volume within the set of all states.

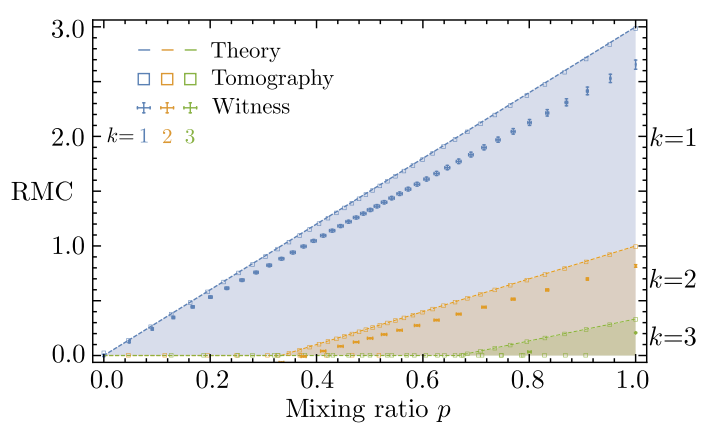

FIG. 4. Measuring multilevel coherence. The plot shows experimentally measured robustness of $(k+1)$-level coherence for a four-dimensional, noisy maximally coherent state $\rho(p)$ as a function of $p \in[0,1]$. The solid lines represent the theory predictions from Eq. (5), and the shaded areas indicate the regions where multilevel coherence for $k=1$ (blue), $k=2$ (orange), $k=3$ (green) can be observed. The open squares correspond to the robustness of $(k+1)$-level coherence estimated from SDP in Eq. (11) applied to the experimentally reconstructed density matrices. The $5 \sigma$ statistical confidence regions obtained from Monte Carlo resampling are on the order of $10^{-3}$ for $p$ and on the order of $10^{-2}$ for the RMC. These are smaller than the symbol size and thus not shown. The data points with error bars correspond to the absolute values of the negative expectation values of $W_{k}\left(\psi_{4}^{+}\right)$in Eq. (9), which provide a lower bound on the RMC. 


\section{Necessary and sufficient criteria for coherence beyond two levels}

Given a $d \times d$ matrix $A$, the associated comparison matrix is defined as (see Definition 2.5.10 of Ref. [58])

$$
M(A)_{i j}=\left\{\begin{array}{cc}
\left|A_{i i}\right| & \text { if } i=j \\
-\left|A_{i j}\right| & \text { if } i \neq j .
\end{array}\right.
$$

We refer the reader to Sec. 2.5 of Ref. [58] for more details on the many properties of this construction. We now present our result on the full characterization of the set $C_{2}$ in arbitrary dimensions, whose proof is given in the Supplemental Material [41].

Theorem 1. A density matrix $\rho$ is such that $n_{C}(\rho) \leq 2$ if and only if $M(\rho) \geq 0$ in the sense of positive semidefiniteness.

An easy corollary of the above result is a simple rule to completely classify qutrit states according to their coherence number. Namely, a qutrit state $\rho$ has coherence number at most 2 if $\operatorname{det} M(\rho) \geq 0$, and 3 otherwise [41].

\section{Necessary conditions for multilevel coherence}

As indicated in Fig. 1(a), the set of fully incoherent states $C_{1}$ has zero volume within $\mathcal{D}(\mathcal{H})$ [9]. This has the important consequence that a state generated randomly will practically never be fully incoherent and that arbitrarily small perturbations applied to a fully incoherent state will create coherence [59]. In other words, under realistic experimental conditions, one cannot prepare or verify a fully incoherent state. In contrast, we show in the Supplemental Material [41] that the sets $C_{k}$ are always of nonzero volume for any $k \geq 2$, and we thus present a rich and experimentally meaningful hierarchy within $\mathcal{D}(\mathcal{H})$, as shown in Fig. 1(a).

Specifically, we have that, if a state $\rho$ satisfies

$$
\rho \geq \frac{d-k}{d-1} \Delta(\rho),
$$

with $\Delta$ the fully decohering map, then $\rho \in C_{k}$. Furthermore, a corollary of Theorem 1 is that if a state $\rho$ satisfies

$$
\operatorname{Tr}\left(\rho^{2}\right) \leq \frac{1}{d-1},
$$

then such a state cannot have multilevel coherence; i.e., $\rho \in C_{2}$ for any reference basis. Observe that the condition (8) is equivalent to being close enough to the maximally mixed state $\mathbb{I} / d[41]$ and that the upper bound in Eq. (8) is tight, as it is achieved by states at the boundary of the set of density matrices, e.g., by $\rho=(\mathbb{I}-|\psi\rangle\langle\psi|) /(d-1)$, with $|\psi\rangle$ any arbitrary pure state [41]. This corollary can be considered the correspondent in coherence theory of the celebrated fact, in entanglement theory, that there is a ball of (fully) separable states surrounding the maximally mixed state [60-62].

\section{Witnessing multilevel coherence}

In analogy to the parallel concept for quantum entanglement, we introduce an efficient alternative to the tomographic approach: multilevel coherence witnesses. In the following, we denote by $\lambda^{\min }(X)$ and $\lambda^{\max }(X)$ the smallest and largest eigenvalues of a Hermitian operator or matrix $X=X^{\dagger}$, respectively.

Since the sets $C_{k}$ are convex, for any $\rho \notin C_{k}$ there exists a $(k+1)$-level coherence witness $W$ such that $\operatorname{Tr}(W \rho)<0$ and $\operatorname{Tr}(W \sigma) \geq 0$ for all $\sigma \in C_{k}$ [63]. A negative expectation value for $W$ thus certifies the $(k+1)$-level coherence of $\rho$ in a single measurement.

Given any pure state $|\psi\rangle=\sum_{i=1}^{d} c_{i}|i\rangle \in \mathcal{H}$, one can construct a $(k+1)$-level coherence witness as

$$
W_{k}(\psi)=\mathbb{I}-\frac{1}{\sum_{i=1}^{k}\left|c_{i}^{\downarrow}\right|^{2}}|\psi\rangle\langle\psi|,
$$

where $c_{i}^{\downarrow}$ are the coefficients $c_{i}$ rearranged into nonincreasing modulus order. This construction ensures that $\langle\phi|W| \phi\rangle \geq 0$ for all $|\phi\rangle$ with $r_{C}(\phi) \leq k$ since $\max _{r_{C}(\phi) \leq k}|\langle\phi \mid \psi\rangle|^{2}=\sum_{i=1}^{k}\left|c_{i}^{\downarrow}\right|^{2}$ [41]. On the other hand, it is clear that $W_{k}(\psi)$ always reveals the $k+1$ coherence of $|\psi\rangle$ if present since $\left\langle\psi\left|W_{k}(\psi)\right| \psi\right\rangle=1-\left(\sum_{i=1}^{k}\left|c_{i}^{\downarrow}\right|^{2}\right)^{-1}$, which is negative if $|\psi\rangle\langle\psi| \notin C_{k}$. For the maximally coherent state $\left|\psi_{d}^{+}\right\rangle$, we then find $W_{k}\left(\psi_{d}^{+}\right)=\mathbb{I}-\frac{d}{k}\left|\psi_{d}^{+}\right\rangle\left\langle\psi_{d}^{+}\right|$.

More generally, the set $C_{k}^{*}$ of $(k+1)$-level coherence witnesses is obtained as the dual of the set $C_{k}$ and is characterized by the following theorem, proved in the Supplemental Material [41].

Theorem 2. A self-adjoint operator $W$ is in $C_{k}^{*}$ if and only if

$$
P_{I} W P_{I} \geq 0 \quad \forall I \in \mathcal{P}_{k},
$$

where $\mathcal{P}_{k}$ is the set of all the $k$-element subsets of $\{1,2, \ldots, d\}$, and $P_{I}:=\sum_{i \in I}|i\rangle\langle i|$.

Hence, verifying that a given self-adjoint operator $W$ is a $(k+1)$-level coherence witness requires verifying the positive semidefiniteness of all $(k \times k)$-dimensional principal submatrices of the matrix representation of $W$ with respect to the classical basis.

We observe that, while nontrivial multilevel-coherence witnesses necessarily have negative eigenvalues, the number of such negative eigenvalues is severely constrained [41]. In particular, we have the following observation.

Observation 1. A $(k+1)$-level coherence witness $W_{k} \in C_{k}^{\star}$ has at most $d-k$ negative eigenvalues. All the eigenvalues are bounded from below by $-(d-k / k) \lambda^{\max }\left(W_{k}\right)$. 
It is worth remarking that the eigenvector corresponding to the single negative eigenvalue of a $d$-level-coherence witness (that is, $k=d-1$ ) must itself exhibit $d$-level coherence.

The characterization of multilevel-coherence witnesses of Theorem 2 finds explicit application in the dual form of the SDP formulation of the RMC [54]. In the case of RMC, strong duality holds, which means that the primal and dual forms of the problem are equivalent, with the latter given by

$$
\begin{array}{cc}
R_{C_{k}}(\rho)=\max & -\operatorname{Tr}(\rho W) \\
\text { s.t. } & P_{I} W P_{I} \geq 0 \quad \forall I \in \mathcal{P}_{k} \\
& W \leq \mathbb{I} .
\end{array}
$$

Hence, while a lower bound on $R_{C_{k}}(\rho)$ can be obtained from the negative expectation value of any observable $W \in C_{k}^{\star}$ such that $W \leq \mathbb{I}$, the dual SDP for the RMC actually computes an optimal $(k+1)$-level coherence witness whose expectation value matches $R_{C_{k}}(\rho)$.

For the family of noisy maximally coherent states $\rho(p)$, the witness $W_{k}\left(\psi_{d}^{+}\right)$of Eq. (9) turns out to be optimal, independently of the noise parameter $p$, and we calculate $\operatorname{Tr}\left(W_{k}\left(\psi_{d}^{+}\right) \rho(p)\right)=(1 / k)[(k-1)-p(d-1)]$. Figure 4 shows the absolute value of the experimentally obtained (negative) expectation values of $W_{k}\left(\psi_{4}^{+}\right)$for a range of values of $p$. This demonstrates that multilevel coherence can be quantitatively witnessed in the laboratory using only a single measurement. Experimentally, however, implementing the optimal witness requires a projection onto a maximally coherent state, which is very sensitive to noise. Indeed, in our experiment, we observe a small degree of beam steering by the wave plates, leading to phase uncertainty between the basis states $|0,1\rangle$ and $|2,3\rangle$. As a consequence, the witness becomes suboptimal and only provides a lower bound on the RMC of the experimental state. In contrast, our results show that the larger number of measurements in the tomographic approach and the associated maximum likelihood reconstruction add resilience to experimental imperfections.

\section{E. Bounding multilevel coherence}

In practice, one might often not be able to perform full tomography on a system or measure the optimal witness. Remarkably, one can obtain a lower bound on the RMC of an experimentally prepared state $\rho$ from any set of experimental data. Specifically, the SDP in Eq. (S16) in the Supplemental Material [41] computes the minimal RMC of a state $\tau \in \mathcal{D}(\mathcal{H})$ that is consistent with a set of measured expectation values $o_{i}=\operatorname{Tr}\left(O_{i} \rho\right)$ for $n$ observables $\left\{O_{i}\right\}_{i=1}^{n}$ to within experimental uncertainty. This is particularly appealing when one has already performed a set of (well-characterized) measurements and wishes to use these to estimate the multilevel coherence of the input state. Note that $d^{2}-1$ linearly independent observables (assuming vanishingly small errors and not including the identity, which accounts for normalization) are sufficient to uniquely determine the state, in which case we could use the original SDP, Eq. (11). A similar approach can, in principle, be used to bound other quantum properties, like entanglement, from limited data [64], also via the use of SDPs [65]. In the case of entanglement, one still has to deal with the fact that the separability condition is not a simple SDP constraint, which is relevant even in the case of complete information: So, in general, the obstacle constituted by lack of information combines with the obstacle of the difficulty of entanglement detection.

We experimentally estimate the lower bounds from Eq. (S16) in the Supplemental Material [41] for an increasing number of randomly chosen observables $O_{i}$, measured on a four-dimensional maximally coherent state and on a noisy maximally coherent state with $p=$ $0.8874 \pm 0.0007$ (see Fig. 5). The results show that our lower bounds become nontrivial already for a small number of observables and converge to a suboptimal yet highly informative value. The remaining gap of about $5 \%$ between these bounds and the tomographically estimated RMC is due to our conservative $5 \sigma$ error bounds and could be improved by incorporating maximum-likelihood or Bayesian estimation techniques (see Supplemental Material [41] for details). We also find that the number of measurements required for nontrivial bounds increases slowly with the coherence level, and the bounds saturate more quickly for states with more coherence.

We further describe how any single observable $O$ may provide a lower bound to the RMC [41]. Consider witnesses of the form $W=\alpha \mathbb{I}+\beta O$, with $\alpha, \beta$ real coefficients, which

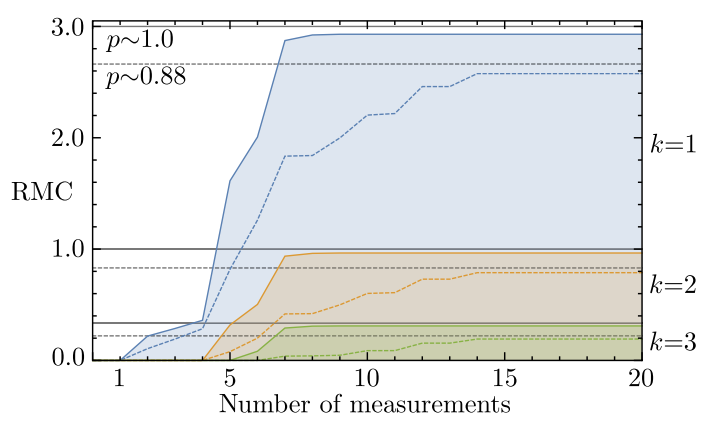

FIG. 5. Bounding multilevel coherence from arbitrary measurements. The blue, orange, and green solid lines correspond to the experimental lower bound on the robustness of multilevel coherence for $k=2,3,4$, respectively, for a maximally coherent state $\left|\psi_{4}^{+}\right\rangle$, while the grey solid lines are the theory prediction. These bounds are obtained from the SDP in Eq. (S16) in the Supplemental Material [41] for an increasing number of randomly chosen projective measurements, taking into account $5 \sigma$ statistical uncertainties. The colored dashed lines correspond to the lower bounds for the noisy maximally coherent state $\rho(0.8874 \pm 0.0007)$ using the same observables, with the grey dashed line being the theory prediction for this state. 
then give a lower bound to the RMC via Eq. (11). Define the $k$-coherence numerical range of $O$ as the interval $\mathrm{NR}_{C_{k}}(O)=\left\{\operatorname{Tr}\left(O \sigma_{C_{k}}\right): \sigma \in C_{k}\right\}$ (the case $k=d-1$ was studied in [66,67]) and define its extreme points $\lambda_{C_{k}}^{\min }(O)=$ $\operatorname{minNR}_{C_{k}}(O)$ and $\lambda_{C_{k}}^{\max }(O)=\operatorname{maxNR}_{C_{k}}(O)$. Notice that $\lambda_{C_{k}}^{\min }(O)=\min _{r_{C}(|\psi\rangle) \leq k}\langle\psi|O| \psi\rangle=\min _{I \in \mathcal{P}_{k}} \lambda^{\min }\left(P_{I} O P_{I}\right)$ [similarly for $\lambda_{C_{k}}^{\max }(O)$ ]. Notice also that $\mathrm{NR}_{C_{d}}(O)$ is the standard numerical range of $O$, and $\lambda_{C_{d}}^{\min }(O)=\lambda^{\min }(O)$ (similarly for the maximal values). In general, $\mathrm{NR}_{C_{k}}(O) \subseteq$ $\mathrm{NR}_{C_{k^{\prime}}}(O)$ for $k \leq k^{\prime}$. If $\operatorname{Tr}(O \rho) \in \mathrm{NR}_{C_{k}}(O)$, that is, if $\lambda_{C_{k}}^{\min }(O) \leq \operatorname{Tr}(O \rho) \leq \lambda_{C_{k}}^{\max }(O)$, then the expectation value of $O$ is compatible with $\rho$ being in $C_{k}$, and we do not gain any information on $R_{C_{k}}(\rho)$. If instead $\operatorname{Tr}(O \rho)>\lambda_{C_{k}}^{\max }(O)$ or $\operatorname{Tr}(O \rho)<\lambda_{C_{k}}^{\min }(O)$, the following bound is nontrivial:

$R_{C_{k}}(\rho) \geq \max \left\{0, \frac{\operatorname{Tr}(O \rho)-\lambda_{C_{k}}^{\max }(O)}{\lambda_{C_{k}}^{\max }(O)-\lambda^{\min }(O)}, \frac{\lambda_{C_{k}}^{\min }(O)-\operatorname{Tr}(O \rho)}{\lambda^{\max }(O)-\lambda_{C_{k}}^{\min }(O)}\right\}$.

Notice that the lower bound is monotonically nonincreasing with $k$.

\section{F. Multilevel coherence as a resource for quantum-enhanced phase discrimination}

To demonstrate the operational significance of multilevel coherence, we show that it is the key resource for the following task, illustrated in Fig. 6(a). Suppose that a physical device can apply one of $n$ possible quantum operations $\left\{\Lambda_{m}\right\}_{m=1}^{n}$ to a quantum state $\rho$, according to the known prior probability distribution $\left\{p_{m}\right\}_{m=1}^{n}$. The output state is then subject to a single generalized measurement with elements $\left\{M_{m}\right\}_{m=1}^{n}$ satisfying $M_{m} \geq 0$ and $\sum_{m=1}^{n} M_{m}=\mathbb{I}$. Our objective is to infer the label $m$ of the quantum operation that was applied.

We now consider a special case of these tasks, known as phase discrimination, which is an important primitive in quantum information processing, featured in optimal cloning, dense coding, and error correction protocols [39,68-70]. Here, the operations imprint a phase on the state through the transformation $\mathcal{U}_{\phi_{m}}(\rho):=U_{\phi_{m}} \rho U_{\phi_{m}}^{\dagger}$, where $U_{\phi_{m}}:=\exp \left(-i H \phi_{m}\right)$ is generated by the Hamiltonian $H=\sum_{j=0}^{d-1} j|j\rangle\langle j|$. The probability of success for inferring the label $m$ in the task specified by $\Theta=$ $\left\{\left(p_{m}, \phi_{m}\right)\right\}_{m=1}^{n}$ is then

$$
p_{\text {succ }}^{\Theta}(\rho):=\sum_{m=1}^{n} p_{m} \operatorname{Tr}\left(\mathcal{U}_{\phi_{m}}(\rho) M_{m}\right) .
$$

Since the Hamiltonian is diagonal in the classical basis and leaves fully incoherent states invariant, the strategy that maximizes $p_{\text {succ }}^{\Theta}$ while at the same time only making use of incoherent states is to guess the most likely label, that is, to take $M_{m}=\mathbb{I} \delta_{m, m_{\max }}$, succeeding with probability $p_{\max }^{\Theta}:=$ $p_{m_{\max }}=\max \left\{p_{m}\right\}_{m=1}^{n}$. On the other hand, a probe state $\rho$ (a)

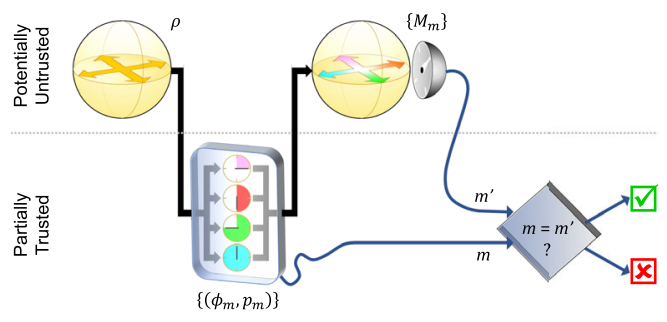

(b)

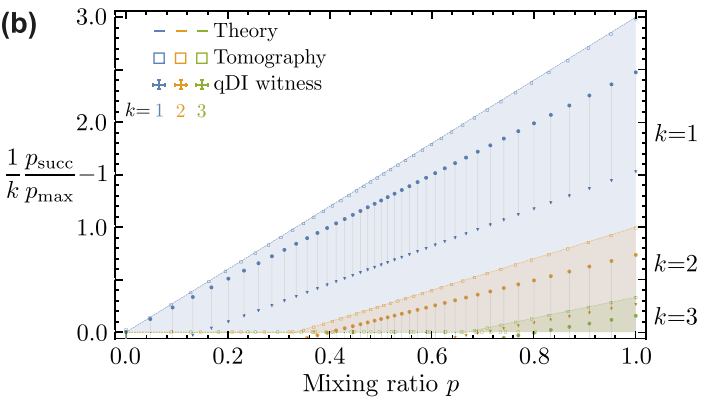

FIG. 6. Semi-device-independent witnessing of multilevel coherence. (a) A $d$-dimensional probe state $\rho$ is sent into a black box, which imprints one of $n$ phases $\left\{\phi_{m}\right\}_{m=1}^{n}$ onto the state at random according to the prior probability distribution $\left\{p_{m}\right\}_{m=1}^{n}$. To infer the index $m$ of the imprinted phase, the state is then subjected to a single generalized measurement with elements $\left\{M_{m}\right\}_{m=1}^{n}$, yielding outcome $m^{\prime}$. This strategy succeeds, i.e., $m^{\prime}=m$, with probability $p_{\text {succ }}^{\Theta}(\rho)$, given by Eq. (13), which exceeds the optimal classical success probability $p_{\max }:=\max \left\{p_{m}\right\}_{m=1}^{n}$ by a factor greater than $k$ only when $(k+1)$-level coherence is present in the initial state $\rho$. Since evaluating the probability of success can be done without any information about the measurement device, based only on the assumption that incoherent states are unchanged by the black box, this scheme provides a semi-device-independent method to witness and estimate the robustness of multilevel coherence in the probe. (b) The experimentally measured bounds on the robustness of $(k+1)$-level coherence from the performance of noisy maximally coherent states $\rho(p)$ in the phase discrimination task $\tilde{\Theta}$, as a function of $p \in[0,1]$. Plot as in Fig. 4, where solid lines represent the theory predictions, and open squares are the measured RMC from quantum-state tomography for comparison. The filled circles (higher) correspond to the semi-device-independent witness as discussed in the text, under the assumption that the application of the phases leaves incoherent states invariant. An upside-down triangle (lower) corresponds to each filled circle, which represents the conservative estimate of multilevel coherence obtained from the phase discrimination task by taking into account experimental imperfections in the implementation of the unitaries (see Supplemental Material [41] for details). The gray lines connecting circles and the corresponding triangles serve as a visual aid. For all data, $5 \sigma$ statistical confidence regions obtained from Monte Carlo resampling are on the order of $10^{-3}$ for $p$ and on the order of $10^{-2}$ for the RMC. 
with nonzero coherence can outperform this strategy $[8,9]$. Here, we find that genuine $(k+1)$-level coherence is necessary for $p_{\text {succ }}^{\Theta}(\rho)$ to achieve a better than $k$-fold enhancement over $p_{\max }^{\Theta}$ in any phase discrimination task $\theta$.

Theorem 3. For any phase discrimination task $\Theta$ and any probe state $\rho$,

$$
\frac{p_{\mathrm{succ}}^{\Theta}(\rho)}{p_{\max }^{\Theta}} \leq k\left(1+R_{C_{k}}(\rho)\right) .
$$

This theorem is proved in the Supplemental Material [41], where we also show that for the specific task $\tilde{\Theta}=$ $\{[(1 / d),(2 \pi m / d)]\}_{m=1}^{d}$ of discriminating $d$ uniformly distributed phases and for a noisy maximally coherent probe, the bound in Eq. (14) becomes tight. This demonstrates the key role of genuine multilevel coherence as a necessary ingredient for quantum-enhanced phase discrimination, unveiling a hierarchical resource structure that goes significantly beyond previous studies that were only concerned with the coarse-grained description of coherence $[8,9]$.

Note that this provides an operational significance to the robustness of multilevel coherence in addition to its operational significance in terms of resilience of noise, which in turn can be thought of also in geometric terms.

\section{Semi-device-independent witnessing of multilevel coherence}

An important consequence of Eq. (14) is that whenever $p_{\text {succ }}^{\Theta}(\rho) / p_{\max }^{\Theta}>k$, the probe state $\rho$ must have $(k+1)$ level coherence. Consequently, the performance of an unknown state $\rho$ in any phase discrimination task $\Theta$ provides a witness of genuine multilevel quantum coherence. We remark that the success probability for an arbitrary quantum state can be evaluated without any knowledge of the devices used-neither the one imprinting the phase nor the final measurement. Evaluating the witness only relies on the fact that $p_{\text {succ }}^{\Theta}(\rho) \leq p_{\max }^{\Theta}$ for any $\rho \in C_{1}$, which in turn relies on $\mathcal{U}_{\phi_{m}}(\rho)=\rho$ for any $\rho \in C_{1}$. In other words, under the condition that no information is imprinted on incoherent states, the witness can be evaluated without any additional knowledge of the devices used. We conclude that phase discrimination, as demonstrated in this paper, is a semi-device-independent approach to measure multilevel coherence as quantified by the RMC.

Figure 6 shows our experimental results for semi-deviceindependent witnessing of multilevel coherence using the phase discrimination task $\tilde{\Theta}$ for a range of noisy maximally coherent states, also taking into account experimental imperfections when it comes to the hypothesis $\mathcal{U}_{\phi_{m}}(\rho)=$ $\rho$ for any $\rho \in C_{1}$ (see Supplemental Material [41]). As with any witnessing approach, this method, in general, only provides lower bounds on the RMC, yet in contrast to the optimal multilevel witness measured in Fig. 4, the present approach does not rely on any knowledge of the measurements used.

\section{DISCUSSION}

The study of genuine multilevel coherence is pivotal, not only for fundamental questions but also for applications ranging from transfer phenomena in many-body and complex systems to quantum technologies, including quantum metrology and quantum communication. In particular, for verifying that a quantum device is working in a nonclassical regime, it is crucial to certify and quantify multilevel coherence with as few assumptions as possible. Our metrological approach satisfies these criteria by making it possible to verify the preparation of large superpositions and discriminate between them, using only the ability to apply phase transformations that leave incoherent states (approximately) invariant. The goal of the phasediscrimination task we consider is to distinguish a finite number of phases in a single-shot scenario, and the figure of merit we adopt is the probability of success of correctly identifying the phase imprinted onto the input state. In particular, given our figure of merit, there is no notion of "closeness" of the guess to the actual phase. In contrast, in sensing applications, the task is often to measure an unknown phase with high precision [71], a task we refer to as "phase estimation." For the latter, the figure of merit is the uncertainty of the estimate, and superpositions of the kind $(|1\rangle+|d\rangle) / \sqrt{2}$, that is, involving eigenstates of the observable that correspond to the largest gap in eigenvalues, can be argued to be optimal [72]. When dealing with phase estimation, the relevant notion is that of unspeakable coherence (or asymmetry) [4], and which eigenstates are superposed is very important. On the other hand, for the kind of phase-discrimination task we consider, genuine multilevel coherence of a state like $(|1\rangle+|2\rangle+\cdots+$ $|d\rangle) / \sqrt{d}$ plays a key role. While it was already known that such a maximally coherent state provides the best performance in discriminating equally spaced phases [73], here we find that the robustness of multilevel coherence of a generic mixed state captures its usefulness in a generic phase discrimination task. This allows us to reverse the argument and use such usefulness to certify multilevel coherence in a semi-device-independent way.

Our analysis of coherence rank and number, multilevel coherence witnesses, and robustness uses and adapts notions originally studied in the context of entanglement theory [28] and hence provides further parallels between the resource theories of quantum coherence and entanglement, whose interplay is attracting substantial interest [1]. However, a notable difference between the two that we find, emphasize, and exploit is that multilevel coherence, unlike entanglement, can be characterized and quantified via semidefinite programming rather than general convex optimization [50]. This highlights multilevel coherence as a powerful, yet experimentally accessible quantum resource.

Remarkably, we show that is it possible to use the notion of the comparison matrix to devise a test that faithfully 
detects genuine three-level coherence and above. We expect such a result to find widespread application in the study of coherence, both theoretically and experimentally. As two immediate applications, we were able to provide a full analytical classification of multilevel coherence for a qutrit, as well as to prove the existence of a ball (actually, the largest possible one, in the Hilbert-Schmidt norm) around the maximally mixed state that contains states that do not exhibit genuine multilevel coherence. This parallels the celebrated result, in entanglement theory, that there is a ball of fully separable states around the maximally mixed state of a multipartite system, and it explicitly shows that generating genuine multilevel coherence is a nontrivial experimental task.

It is worth remarking that a number of our results also apply in the case of infinite-dimensional systems, such as a harmonic oscillator or quantized field. Indeed, one can always consider, e.g., the quantum (multilevel) coherence exhibited by a system among a subset of states of the incoherent basis, which then provides a bound on the (multilevel) coherence in the entire Hilbert space of the system.

Finally, our work triggers several questions to stimulate further research. These include conceptual questions regarding the exact (geometric) structure and volume of the sets $C_{k}$, and how sets $C_{k}$ and $C_{l}^{\prime}$ defined with respect to different classical bases intersect, the best further use of tools like the comparison matrix to detect and quantify multilevel coherence, or general purity-based bounds on multilevel coherence. From a more practical point of view, a natural question is how to best choose a finite set of observables to estimate the multilevel coherence of the state of a system, for example, via the SDP in the Supplemental Material [41]. This is particularly important when one has limited access to the system under observation, as in a biological setting $[30,74,75]$. Independently of the particular choice of observables, our work provides a plethora of readily applicable tools to facilitate the detection, classification, and quantitative estimation of quantum coherence phenomena in systems of potentially large complexity with minimum assumptions, paving the way towards a deeper understanding of their functional role. Further theoretical investigation and experimental progress along these lines may lead to fascinating insights and advances in other branches of science where the detection and exploitation of (multilevel) quantum coherence is or can be of interest.

\section{ACKNOWLEDGMENTS}

We thank M. B. Plenio, B. Regula, V. Scarani, and A. Streltsov for helpful discussions and T. Vulpecula for experimental assistance. This work was supported in part by the Centres for Engineered Quantum Systems (CE110001013) and for Quantum Computation and Communication Technology (CE110001027), the Engineering and Physical Sciences Research Council
(Grant No. EP/N002962/1), and the Templeton World Charity Foundation (TWCF 0064/AB38). A. F. acknowledges the Royal Society for support via a Theo Murphy Blue Skies award. We acknowledge financial support from the European Union's Horizon 2020 Research and Innovation Programme under the Marie Skłodowska-Curie Action OPERACQC (Grant Agreement No. 661338) and the ERC Starting Grant GQCOP (Grant Agreement No. 637352), and from the Foundational Questions Institute under the Physics of the Observer Programme (Grant No. FQXi-RFP-1601).

[1] A. Streltsov, G. Adesso, and M. B. Plenio, Colloquium: Quantum Coherence as a Resource, Rev. Mod. Phys. 89, 041003 (2017).

[2] J. Aberg, Quantifying Superposition, arXiv:quant-ph/ 0612146.

[3] T. Baumgratz, M. Cramer, and M. Plenio, Quantifying Coherence, Phys. Rev. Lett. 113, 140401 (2014).

[4] I. Marvian and R. W. Spekkens, How to Quantify Coherence: Distinguishing Speakable and Unspeakable Notions, Phys. Rev. A 94, 052324 (2016).

[5] B. Coecke, T. Fritz, and R. W. Spekkens, A Mathematical Theory of Resources, Inf. Comput. 250, 59 (2016).

[6] M. Horodecki and J. Oppenheim, (Quantumness in the Context) Resource Theories, Int. J. Mod. Phys. B 27, 1345019 (2013).

[7] F. G. Brandão and G. Gour, Reversible Framework for Quantum Resource Theories, Phys. Rev. Lett. 115, 070503 (2015).

[8] C. Napoli, T. R. Bromley, M. Cianciaruso, M. Piani, N. Johnston, and G. Adesso, Robustness of Coherence: An Operational and Observable Measure of Quantum Coherence, Phys. Rev. Lett. 116, 150502 (2016).

[9] M. Piani, M. Cianciaruso, T. R. Bromley, C. Napoli, N. Johnston, and G. Adesso, Robustness of Asymmetry and Coherence of Quantum State, Phys. Rev. A 93, 042107 (2016).

[10] X. Yuan, H. Zhou, Z. Cao, and X. Ma, Intrinsic Randomness as a Measure of Quantum Coherence, Phys. Rev. A 92, 022124 (2015).

[11] A. Winter and D. Yang, Operational Resource Theory of Coherence, Phys. Rev. Lett. 116, 120404 (2016).

[12] T. Biswas, M. García Díaz, and A. Winter, Interferometric Visibility and Coherence, Proc. R. Soc. A 473, 0170 (2017).

[13] A. Streltsov, U. Singh, H. S. Dhar, M. N. Bera, and G. Adesso, Measuring Quantum Coherence with Entanglement, Phys. Rev. Lett. 115, 020403 (2015).

[14] E. Chitambar, A. Streltsov, S. Rana, M. Bera, G. Adesso, and M. Lewenstein, Assisted Distillation of Quantum Coherence, Phys. Rev. Lett. 116, 070402 (2016).

[15] G. Adesso, T. R. Bromley, and M. Cianciaruso, Measures and Applications of Quantum Correlations, J. Phys. A 49 , 473001 (2016).

[16] I. Marvian, R. W. Spekkens, and P. Zanardi, Quantum Speed Limits, Coherence, and Asymmetry, Phys. Rev. A 93, 052331 (2016). 
[17] D. Girolami, Observable Measure of Quantum Coherence in Finite Dimensional Systems, Phys. Rev. Lett. 113, 170401 (2014).

[18] H. Ren, A. Lin, S. He, and X. Hu, Quantitative Coherence Witness for Finite Dimensional States, Ann. Phys. (Berlin) 387, 281 (2017).

[19] P. Kammerlander and J. Anders, Coherence and Measurement in Quantum Thermodynamics, Sci. Rep. 6, 22174 (2016).

[20] R. Uzdin, A. Levy, and R. Kosloff, Equivalence of Quantum Heat Machines, and Quantum-Thermodynamic Signatures, Phys. Rev. X 5, 031044 (2015).

[21] M. Hillery, Coherence as a Resource in Decision Problems: The Deutsch-Jozsa Algorithm and a Variation, Phys. Rev. A 93, 012111 (2016).

[22] P. Giorda and M. Allegra, Coherence in Quantum Estimation, J. Phys. A 51, 025302 (2018).

[23] C. Zhang, B. Yadin, Z.-B. Hou, H. Cao, B.-H. Liu, Y.-F. Huang, R. Maity, V. Vedral, C.-F. Li, G.-C. Guo, and D. Girolami, Detecting Metrologically Useful Asymmetry and Entanglement by a Few Local Measurements, Phys. Rev. A 96, 042327 (2017).

[24] D. Braun, G. Adesso, F. Benatti, R. Floreanini, U. Marzolino, M. W. Mitchell, and S. Pirandola, QuantumEnhanced Measurements without Entanglement, Rev. Mod. Phys. 90, 035006 (2018).

[25] F. Levi and F. Mintert, A Quantitative Theory of Coherent Delocalization, New J. Phys. 16, 033007 (2014).

[26] J. Sperling and W. Vogel, Convex Ordering and Quantification of Quantumness, Phys. Scr. 90, 074024 (2015).

[27] N. Killoran, F. E. Steinhoff, and M. B. Plenio, Converting Nonclassicality into Entanglement, Phys. Rev. Lett. 116, 080402 (2016).

[28] R. Horodecki, P. Horodecki, M. Horodecki, and K. Horodecki, Quantum Entanglement, Rev. Mod. Phys. 81, 865 (2009).

[29] O. Gühne and G. Tóth, Entanglement Detection, Phys. Rep. 474, 1 (2009).

[30] C.-M. Li, N. Lambert, Y.-N. Chen, G.-Y. Chen, and F. Nori, Witnessing Quantum Coherence: From Solid-State to Biological Systems, Sci. Rep. 2, 885 (2012).

[31] M. Lostaglio, D. Jennings, and T. Rudolph, Description of Quantum Coherence in Thermodynamic Processes Requires Constraints Beyond Free Energy, Nat. Commun. 6, 6383 (2015).

[32] G. D. Scholes, G. R. Fleming, L. X. Chen, A. AspuruGuzik, A. Buchleitner, D. F. Coker, G. S. Engel, R. van Grondelle, A. Ishizaki, D. M. Jonas, J. S. Lundeen, J. K. McCusker, S. Mukamel, J. P. Ogilvie, A. Olaya-Castro, M. A. Ratner, F. C. Spano, K. B. Whaley, and X. Zhu, Using Coherence to Enhance Function in Chemical and Biophysical Systems, Nature (London) 543, 647 (2017).

[33] B. Witt and F. Mintert, Stationary Quantum Coherence and Transport in Disordered Networks, New J. Phys. 15, 093020 (2013).

[34] T. Scholak, F. de Melo, T. Wellens, F. Mintert, and A. Buchleitner, Efficient and Coherent Excitation Transfer Across Disordered Molecular Networks, Phys. Rev. E 83, 021912 (2011).
[35] M. Tiersch, S. Popescu, and H. J. Briegel, A Critical View on Transport and Entanglement in Models of Photosynthesis, Phil. Trans. R. Soc. A 370, 3771 (2012).

[36] S. Chin, Generalized Coherence Concurrence and Path Distinguishability, J. Phys. A 50, 475302 (2017).

[37] B. Regula, M. Piani, M. Cianciaruso, T. R. Bromley, A. Streltsov, and G. Adesso, Converting Multilevel Nonclassicality into Genuine Multipartite Entanglement, New J. Phys. 20, 033012 (2018).

[38] S. Chin, Coherence Number as a Discrete Quantum Resource, Phys. Rev. A 96, 042336 (2017).

[39] D. Gottesman, in Quantum Computing and Quantum Communications (Springer, New York, 1999), pp. 302-313.

[40] S. M. Barnett and S. Croke, Quantum State Discrimination, Adv. Opt. Photonics 1, 238 (2009).

[41] See Supplemental Material at http://link.aps.org/ supplemental/10.1103/PhysRevX.8.041007 for details on the derivations and proofs.

[42] B. M. Terhal and P. Horodecki, Schmidt Number for Density Matrices, Phys. Rev. A 61, 040301 (2000).

[43] A. S. Sørensen and K. Mølmer, Entanglement and Extreme Spin Squeezing, Phys. Rev. Lett. 86, 4431 (2001).

[44] M. Nielsen and I. Chuang, Quantum Computation and Quantum Information (Cambridge University Press, Cambridge, England, 2010).

[45] G. Vidal and R. Tarrach, Robustness of Entanglement, Phys. Rev. A 59, 141 (1999).

[46] M. Steiner, Generalized Robustness of Entanglement, Phys. Rev. A 67, 054305 (2003).

[47] M. Piani and J. Watrous, Necessary and Sufficient Quantum Information Characterization of Einstein-Podolsky-Rosen Steering, Phys. Rev. Lett. 114, 060404 (2015).

[48] J. Geller and M. Piani, Quantifying Non-Classical and Beyond-Quantum Correlations in the Unified Operator Formalism, J. Phys. A 47, 424030 (2014).

[49] A. W. Harrow and M. A. Nielsen, Robustness of Quantum Gates in the Presence of Noise, Phys. Rev. A 68, 012308 (2003).

[50] F. G. Brandao, Quantifying Entanglement with Witness Operators, Phys. Rev. A 72, 022310 (2005).

[51] F. G. Brandao and R. O. Vianna, Witnessed Entanglement, Int. J. Quantum. Inform. 04, 331 (2006).

[52] K. Bu, N. Anand, and U. Singh, Asymmetry and Coherence Weight of Quantum States, Phys. Rev. A 97, 032342 (2018).

[53] G. Chiribella and D. Ebler, Optimal Quantum Networks and One-Shot Entropies, New J. Phys. 18, 093053 (2016).

[54] S. Boyd and L. Vandenberghe, Convex Optimization (Cambridge University Press, Cambridge, England, 2004).

[55] L. Vandenberghe and S. Boyd, Semidefinite Programming, SIAM Rev. 38, 49 (1996).

[56] J. Watrous, Simpler Semidefinite Programs for Completely Bounded Norms, Chicago J. Theor. Comput. Sci. 2013, 8 (2013).

[57] M. Ringbauer, B. Duffus, C. Branciard, E. G. Cavalcanti, A. G. White, and A. Fedrizzi, Measurements on the Reality of the Wavefunction, Nat. Phys. 11, 249 (2015).

[58] R. A. Horn and C. R. Johnson, Topics in Matrix Analysis (Cambridge University Press, Cambridge, England, 1994). 
[59] A. Ferraro, L. Aolita, D. Cavalcanti, F. Cucchietti, and A. Acin, Almost All Quantum States have Nonclassical Correlations, Phys. Rev. A 81, 052318 (2010).

[60] K. Zyczkowski, P. Horodecki, A. Sanpera, and M. Lewenstein, Volume of the Set of Separable States, Phys. Rev. A 58, 883 (1998).

[61] L. Gurvits and H. Barnum, Largest Separable Balls Around the Maximally Mixed Bipartite Quantum State, Phys. Rev. A 66, 062311 (2002).

[62] L. Gurvits and H. Barnum, Separable Balls Around the Maximally Mixed Multipartite Quantum States, Phys. Rev. A 68, 042312 (2003).

[63] W. Rudin, Functional Analysis (McGraw Hill, New York, 1991).

[64] O. Gühne, M. Reimpell, and R. F. Werner, Estimating Entanglement Measures in Experiments, Phys. Rev. Lett. 98, 110502 (2007).

[65] J. Eisert, F. G. S. L. Brandão, and K. M. R. Audenaert, Quantitative Entanglement Witnesses, New J. Phys. 9, 46 (2007).

[66] C. R. Johnson, Numerical Ranges of Principal Submatrices, Linear Algebra Appl. 37, 23 (1981).
[67] C. R. Johnson and H. A. Robinson, Eigenvalue Inequalities for Principal Submatrices, Linear Algebra Appl. 37, 11 (1981).

[68] N. J. Cerf, Asymmetric Quantum Cloning in Any Dimension, J. Mod. Opt. 47, 187 (2000).

[69] T. Hiroshima, Optimal Dense Coding with Mixed State Entanglement, J. Phys. A 34, 6907 (2001).

[70] R. F. Werner, All Teleportation and Dense Coding Schemes, J. Phys. A 34, 7081 (2001).

[71] V. Giovannetti, S. Lloyd, and L. Maccone, Advances in Quantum Metrology, Nat. Photonics 5, 222 (2011).

[72] V. Giovannetti, S. Lloyd, and L. Maccone, Quantum Metrology, Phys. Rev. Lett. 96, 010401 (2006).

[73] Quantum Detection and Estimation Theory, Mathematics in Science and Engineering (Elsevier Science, New York, 1976).

[74] M. Sarovar, A. Ishizaki, G. R. Fleming, and K. B. Whaley, Quantum Entanglement in Photosynthetic Light-Harvesting Complexes, Nat. Phys. 6, 462 (2010).

[75] S. F. Huelga and M. B. Plenio, Vibrations, Quanta and Biology, Contemp. Phys. 54, 181 (2013). 